\title{
Comparison of Two Identification Models Used in Adaptive Control of Continuous-Stirred Tank Reactor
}

\author{
Jiri Vojtesek and Petr Dostal \\ Tomas Bata University in Zlin, Faculty of Applied Informatics, Nad Stranemi 4511, 76005 Zlin, Czech Republic
}

\begin{abstract}
The goal of this paper is to compare two identification methods - continuous-time and discrete-time. The continuous-time identification model is more accurate but not very suitable for on-line identification. This disadvantage was overcome with the use of differential filters. On the other hand, discrete-time identification model has is more suitable for identification but less accurate. Compromise can be found in the delta model as a special type of the discrete-time model parameters of which are related to the sampling period. The adaptive approach is based on the choice of the External Linear Model, parameters of which are identified recursively which satisfies the adaptivity of this system. Proposed control strategy was applied on the mathematical model of the Continuous Stirred-Tank reactor as a typical nonlinear lumped-parameters system used in the industry.
\end{abstract}

\section{Introduction}

The continuous stirred-tank reactor (CSTR) is typical nonlinear equipment used in the chemical and biochemical industry for production of various chemicals, drugs etc. [1].

The modelling and simulation is great tool which helps with the observing of the system's behaviour and designing of the appropriate controller. The mathematical model of CSTR is usually described by the set of nonlinear ordinary differential equations (ODEs) which can be solved mathematically for example by the RungeKutta's method.

The adaptive control [2] used here for the control is one of the approaches used for the nonlinear systems because it produces good control results. Advantage of this method can be found in very good theoretical background and variety of modifications [3].

The approach used here is based on the choice of the External Linear Model (ELM) which describes controlled, originally nonlinear, process in the linear way for example by the discrete or the continuous transfer function (TF) [3]. Parameters of ELM are then identified recursively during the control and parameters of the controller are recomputed according to identified parameters of the system. The advantage of used polynomial synthesis is that it produces the structure and also relations for computing controller's parameters that reflect identified parameters of ELM.

Two identification models with the continuous-time (CT) model [4] and special type of the discrete-time (DT) model called delta/model [5] where discussed here. Parameters of input and output variables are related to the sampling period. It was proved that parameters of the delta model approach to parameters of the CT model for sufficiently small sampling period [6]. This combination of the continuous-time control synthesis with the discrete-time identification is called "Hybrid adaptive control" and some applications can be found for example in [7] and [8].

The recursive least-squares method is used for online identification. This method is widely used because it is easily programmable in standard programming languages at one hand but it produces sufficiently good identification results with various modifications.

All results in this paper are simulations made in the mathematical software Matlab, version 7.0.1.

\section{Continuous Stirred-Tank Reactor}

The system under the consideration is Continuous Stirred-Tank Reactor (CSTR) with so called Van der Vusse reaction inside [9]:

$$
\begin{aligned}
& A \stackrel{k_{1}}{\longrightarrow} B \stackrel{k_{2}}{\longrightarrow} C \\
& 2 A \stackrel{k_{3}}{\longrightarrow} D
\end{aligned}
$$

The mathematical model of this system comes from material and heat balances inside the reactor and results in the set of four nonlinear ordinary differential equations (ODE):

$$
\begin{gathered}
\frac{d c_{A}}{d t}=\frac{q_{r}}{V_{r}}\left(c_{A 0}-c_{A}\right)-k_{1} c_{A}-k_{3} c_{A}^{2} \\
\frac{d c_{B}}{d t}=-\frac{q_{r}}{V_{r}} c_{B}+k_{1} c_{A}-k_{2} c_{B} \\
\frac{d T_{r}}{d t}=\frac{q_{r}}{V_{r}}\left(T_{r 0}-T_{r}\right)-\frac{h_{r}}{\rho_{r} c_{p r}}+\frac{A_{r} U}{V_{r} \rho_{r} c_{p r}}\left(T_{c}-T_{r}\right)
\end{gathered}
$$

\footnotetext{
a Corresponding author: vojtesek@fai.utb.cz
} 


$$
\frac{d T_{c}}{d t}=\frac{1}{m_{c} c_{p c}}\left(Q_{c}+A_{r} U\left(T_{r}-T_{c}\right)\right)
$$

The mathematical model described by the set of ODE (2) - (5) have state variables concentrations $c_{A}, c_{B}$ and temperatures of the reactant $T_{r}$ and the cooling $T_{c}$. This system provides theoretically four input variables - a volumetric flow rate of the reactant, $q_{r}$, a heat removal of the cooling, $Q_{c}$, an input concentration $c_{A 0}$ and an input temperature of the reactant, $T_{r 0}$. The last two are only theoretical and could not be used as an input variable from the practical point of view.

The scheme of this chemical reactor is in Figure 1.

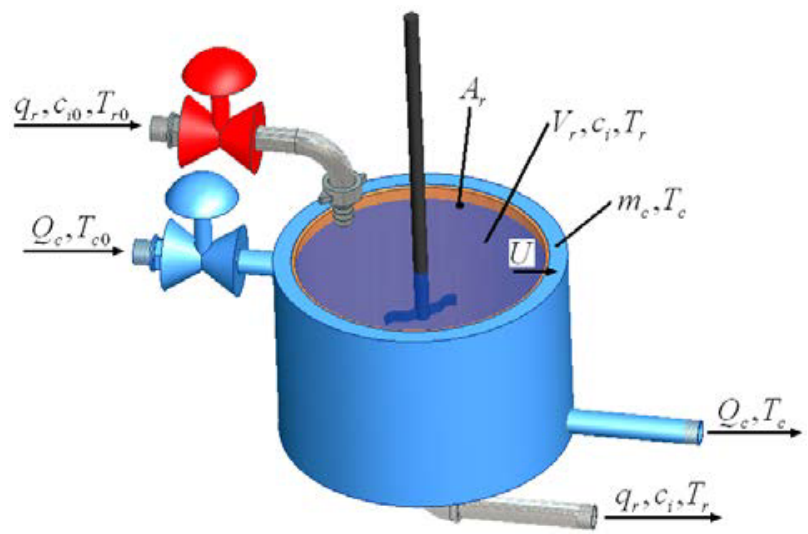

Figure 1. Scheme of the Continuous Stirred-tank Reactor

Due to the simplification of the mathematical model, other variables are supposed to be constant during the control. The volume of the reactor is denoted as $V_{r}, A_{r}$ is the heat exchange surface, $\rho_{r}$ is used for the density of the reactant, $U$ is the heat transfer coefficient, $c_{p c}$ and $c_{p r}$ are specific heat capacities of the cooling and the reactant a $\mathrm{mc}$ is the weight of the cooling mass. Values of these fixed parameters are in Table 1 [9].

Table 1. Fixed parameters of the reactor

\begin{tabular}{|c|c|}
\hline Variable & Value \\
\hline Pre-exp. factor for react. 1 & $k_{01}=2.145 \cdot 10^{10} \mathrm{~min}^{-1}$ \\
\hline Pre-exp. factor for react. 2 & $k_{02}=2.145 \cdot 10^{10} \mathrm{~min}^{-1}$ \\
\hline Pre-exp. factor for react. 3 & $\underline{k}_{03}=1.5072 \cdot 10^{8} \mathrm{~min}^{-1} \mathrm{~mol}^{-1}$ \\
\hline Activation ener. 1 to $\mathrm{R}$ & $E_{1} / R=9758.3 \mathrm{~K}$ \\
\hline Activation ener. 2 to $\mathrm{R}$ & $E_{2} / R=9758.3 \mathrm{~K}$ \\
\hline Activation ener. 3 to $\mathrm{R}$ & $E_{3} / R=8560 \mathrm{~K}$ \\
\hline Enthalpy of reaction 1 & $h_{1}=-4200 \mathrm{~kJ} \cdot \mathrm{kmol}^{-1}$ \\
\hline Enthalpy of reaction 2 & $h_{2}=11000 \mathrm{~kJ} \mathrm{kmol}^{-1}$ \\
\hline Enthalpy of reaction 3 & $h_{3}=41850 \mathrm{~kJ} \mathrm{kmol}^{-1}$ \\
\hline Volume of the reactor & $V_{r}=0.01 \mathrm{~m}^{3}$ \\
\hline Heat capacity of reactant & $c_{p r}=3.01 \mathrm{~kJ} \mathrm{~kg}^{-1} \cdot \mathrm{K}^{-1}$ \\
\hline Heat transfer coefficient & $U=67.2 \mathrm{~kJ} \cdot \mathrm{min}^{-1} \mathrm{~m}^{-2} \mathrm{~K}^{-1}$ \\
\hline Input concentration of $\mathrm{A}$ & $c_{A 0}=5.1 \mathrm{kmol} . \mathrm{m}^{-3}$ \\
\hline Input concentration of $\mathrm{B}$ & $c_{B 0}=0 \mathrm{kmol} . \mathrm{m}^{-3}$ \\
\hline Input temp. of reactant & $T_{r 0}=387.05 \mathrm{~K}$ \\
\hline Density of reactant & $\rho_{r}=934.2 \mathrm{~kg} \cdot \mathrm{m}^{-3}$ \\
\hline Heat capacity of coolant & $c_{p c}=2.0 \mathrm{~kJ} \cdot \mathrm{kg}^{-1} \cdot K^{-1}$ \\
\hline Surface of cooling jacket & $A_{r}=0.215 \mathrm{~m}^{2}$ \\
\hline Weight of coolant & $m_{c}=5 \mathrm{~kg}$ \\
\hline
\end{tabular}

The first step of the simulation is the steady-state analysis which observes behaviour of the system in the steady-state where state variable does not change. This analysis can help us with the choice of the optimal working point for control. Experiments in [8] have shown working point defined by the volumetric flow rate of the reactant $q_{r}^{s}=2.4 \cdot 10^{-3} \mathrm{~m}^{3} \cdot \mathrm{min}^{-1}$ and heat removal of the coolant $Q_{c}^{s}=-18.56 \mathrm{~kJ}^{\mathrm{m}} \mathrm{min}^{-1}$.

The second, dynamic, analysis then observes the behaviour of the system after the step change of the input variable, in this case the heat removal of the coolant, $\Delta Q_{c}$. The observed output is on the other hand the change of the reactant's temperature, $T_{r}$, i.e.

$$
u(t)=\frac{Q_{c}(t)-Q_{c}^{s}}{Q_{c}^{s}} \cdot 100[\%], \quad y(t)=T(t)-T^{s}[K]
$$

The results for various step changes of the input variable from the range $\Delta u(t)=<-100 \% ;+100 \%>$ and results are in Figure 3.

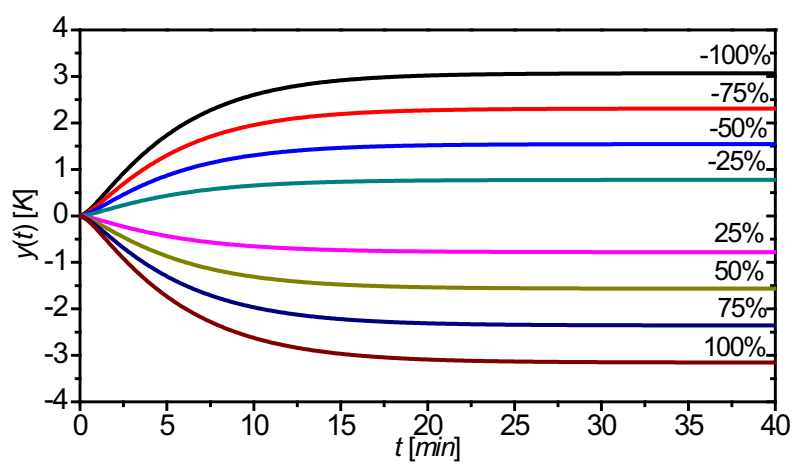

Figure 2. Results of the dynamic analysis for the various changes of the input variable $\Delta u(t)$

Results clearly shows, that all outputs could be described by the second order transfer function (TF) with relative order one in the polynomial form

$$
G(s)=\frac{b(s)}{a(s)}=\frac{b_{1} s+b_{0}}{s^{2}+a_{1} s+a_{0}}
$$

This will be later used for identification in the adaptive control.

\section{Hybrid Adaptive Control}

The control approach here is based on the term "Adaptivity" known from the nature, where plant, animals or even human beings "adapts" their behaviour to the actual environment.

At first we will start with the control synthesis which uses advantages of the polynomial synthesis [10] that satisfies basic control requirements such as stability, disturbance attenuation and reference signal tracking. Moreover, this method produces not only the structure of the controller, but also the relations for computing of controller's parameters.

The simplest, most common known control scheme with one degree of freedom (1DOF) [11] is shown in Figure 3. The controller is here represented by the TF $Q(s)$ and the controlled system is described by TF $G(s)$ from (7). 


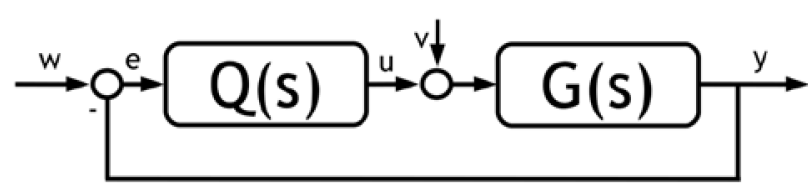

Figure 3. One degree-of-freedom (1DOF) control configuration

The signal $w$ in Figure 3 is reference signal (i.e. wanted value), $v$ denotes disturbance, $u$ is an input and $y$ an output variable. It can be seen, that controller is here only in the feedback part.

The TF of this controller Q(s) is generally:

$$
Q(s)=\frac{q(s)}{p(s)}
$$

where degrees of polynomials $p(s)$ and $q(s)$ must hold properness condition:

$$
\operatorname{deg} q(s) \leq \operatorname{deg} p(s)
$$

The condition for the reference signal tracking is satisfied if the polynomial $\mathrm{p}(\mathrm{s})$ in the denominator of the controller's transfer functions (17) is divided into

$$
p(s)=f(s) \cdot \tilde{p}(s)
$$

where $f(s)$ is a least common divisor of the reference and the disturbance transfer functions. If we have these $\mathrm{TF}$ in the form of the step function, $f(s)=s$ and (17) could be rewritten into

$$
Q(s)=\frac{q(s)}{s \cdot \tilde{p}(s)}
$$

Parameters of controller's polynomials $\tilde{p}(s)$ and $q(s)$ are computed from Diophantine polynomial equation [10]

$$
a(s) \cdot s \cdot \tilde{p}(s)+b(s) \cdot q(s)=d(s)
$$

and they can be solved by the Method of uncertain coefficients. Polynomials $a(s)$ and $b(s)$ in (21) are known from the recursive identification which will be discussed in the next subchapter. The polynomial $d(s)$ on the right side of Diophantine equations (21) is stable optional polynomial which could affect the quality of the control.

Degrees of controller's polynomials $\tilde{p}(s)$ and $q(s)$ and the degree of the stable polynomial $\mathrm{d}(\mathrm{s})$ are

$$
\begin{aligned}
\operatorname{deg} \tilde{p}(s)= & \operatorname{deg} a(s)-1 \quad \operatorname{deg} q(s)=\operatorname{deg} a(s) \\
& \operatorname{deg} d(s)=2 \cdot \operatorname{deg} a(s)
\end{aligned}
$$

As it was already mentioned, polynomial $d(s)$ is optional polynomial which could be designed for example by the Pole-placement method, generally

$$
d(s)=\prod_{i=1}^{\operatorname{deg} d(s)}\left(s+s_{i}\right)
$$

where roots $s_{i}$ are generally in the complex form $s_{i}=\alpha_{i}+\omega_{i} \square j$ and the stability is satisfied for $\alpha_{i}<0$. If we want to obtain an aperiodic output response, $\omega_{i}$ must hold 0 and (14) is then

$$
d(s)=(s+\alpha)^{\operatorname{deg} d}
$$

The equation (15) is very general which could be disadvantage of this method - there is no recommendation for the choice of roots in polynomial $d(s)$. Our previous experiments [8] have shown that is good co connect the choice of this polynomial somehow with the controlled system. The Spectral factorization could be used for this task and it means that the polynomial $d(s)$ is divided into two parts

$$
d(s)=n(s) \cdot(s+\alpha)^{\operatorname{deg} d-\operatorname{deg} n}
$$

where one part is classic pole-placement method and $n(s)$ comes from the Spectral factorization of the polynomial $a(s)$ in the denominator of the controlled system's transfer function (7):

$$
n^{*}(s) \cdot n(s)=a^{*}(s) \cdot a(s)
$$

Advantage of the Spectral factorization can be also find in the feature, that the polynomial $n(s)$ is always stable even if the polynomial $a(s)$ is unstable. This could happen for example by inaccurate estimation at the beginning of the control when an estimator does not have enough information about the system.

\section{Identification Models}

It was already mentioned that the controller is based on the adaptivity. There are several adaptive approaches used in the control theory. The one used here is based on the online recursive identification of the External Linear Model (ELM) of the originally nonlinear system. Parameters of the controller are then recomputed according to identified parameters of the ELM. ELM could be for example TF in the form of (7).

There will be discussed two types of identification models - continuous-time (CT) and discrete-time (DT) in the next subchapters.

\subsection{Continuous-Time Identification Model}

The ELM of the controlled system is described by continuous-time TF $G(s)$ (7) and this relation is also described to the fraction of the the Laplace transform of the output variable, $Y(s)$, to the input variable, $U(s)$, the ELM in the (7) could be also rewritten to the form

$$
a(\sigma) \cdot y(t)=b(\sigma) \cdot u(t)
$$

where $u(t)$ denotes the input variable, $y(t)$ is the output variable and $\sigma$ is the differentiation operator.

The identification of CT model in (18) is problem because the derivatives of the input and the output variables are immeasurable but they could be replaced by the filtered ones denoted by $u_{f}$ and $y_{f}$ and computed from

$$
\begin{aligned}
& c(\sigma) \cdot u_{f}(t)=u(t) \\
& c(\sigma) \cdot y_{f}(t)=y(t)
\end{aligned}
$$

for a new stable polynomial $c(\sigma)$ that fulfils condition $\operatorname{deg} c(\sigma) \geq \operatorname{deg} a(\sigma)$, the Laplace transform of (19) is then

$$
\begin{aligned}
& c(s) \cdot U_{f}(s)=U(s)+o_{1}(s) \\
& c(s) \cdot Y_{f}(s)=Y(s)+o_{2}(s)
\end{aligned}
$$

where polynomials $o_{1}(\mathrm{~s})$ and $o_{2}(\mathrm{~s})$ includes initial conditions of filtered variables. If we substitute (20) into the Laplace transform of the Equation (18), the relation 
for the Laplace transform of the filtered output variable, $Y_{f}(s)$ is

$$
Y_{f}(s)=\frac{b(s)}{a(s)} U_{f}(s)+\Psi(s)
$$

and $\Psi(s)$ is a rational function which contains initial conditions of both filtered and unfiltered variables.

The dynamics of the differential filters $c(s)$ in (20) must be faster than the dynamics of the controlled system [12] which is satisfied if parameters of this polynomial sufficiently small.

The values of filtered values are taken in the discrete time moment $t_{k}=k \cdot T_{v}$ for $k=0,1,2, \ldots N . T_{v}$ is sampling period and the regression vector has $n+m$ parts where $\operatorname{deg} a=n=2$ and $\operatorname{deg} b=m=1$, i.e.

$$
\boldsymbol{\varphi}_{C T}\left(t_{k}\right)=\left[-y_{f}\left(t_{k}\right),-y_{f}^{(1)}\left(t_{k}\right), u_{f}\left(t_{k}\right), u_{f}^{(1)}\left(t_{k}\right)\right]^{T}
$$

The vector of parameters

$$
\boldsymbol{\theta}_{C T}\left(t_{k}\right)=\left[a_{0}, a_{1}, b_{0}, b_{1}\right]^{T}
$$

is computed from the differential equation

$$
y_{f}^{(n)}\left(t_{k}\right)=\boldsymbol{\theta}_{C T}^{T}\left(t_{k}\right) \cdot \boldsymbol{\varphi}_{C T}\left(t_{k}\right)+\Psi\left(t_{k}\right)
$$

where $\Psi$ includes immeasurable errors.

\subsection{Discrete-Time Identification Model}

The discrete-time identification model is better for practical purposes - it is more simple to read input and output variables in the defined time intervals then continuously. We can find also compromise between practically better DT model and more accurate CT model in so called delta-models [8] that are special types of DT models where input and output variables are related to the sampling period.

A new complex variable $\gamma$ is defined generally as [13]

$$
\gamma=\frac{z-1}{\beta \cdot T_{v} \cdot z+(1-\beta) \cdot T_{v}}
$$

for $T_{v}$ as a sampling period and $\beta$ an optional parameter which holds $0 \leq \beta \leq 1$. It is clear, that there could be an infinite number of delta-models as there is modifiability of $\beta$. One of the most used model is Forward delta-model for $\beta=0$ was used here.

The complex variable $\gamma$ is then

$$
\gamma=\frac{z-1}{T_{v}}
$$

It was proved for example in [6], that parameters of the delta-model approaches to the CT ones for sufficiently small sampling period $T_{v}$.

In delta-models, the CT model (18) can be rewritten to the form

$$
a^{\prime}(\delta) y\left(t^{\prime}\right)=b^{\prime}(\delta) u\left(t^{\prime}\right)
$$

where $a^{\prime}(\delta)$ and $b^{\prime}(\delta)$ are discrete polynomials and their coefficients are different from those in CT model but we suppose, that they are close to them because the sampling period is sufficiently small.

The regression vector is in this case for TF (7):

$\boldsymbol{\varphi}_{\delta}(k-1)=\left[-y_{\delta}(k-1),-y_{\delta}(k-2), u_{\delta}(k-1), u_{\delta}(k-2)\right]^{T}(28)$

and the vector of parameters is then

$$
\boldsymbol{\theta}_{\delta}(k)=\left[a_{1}^{\prime}, a_{0}^{\prime}, b_{1}^{\prime}, b_{0}^{\prime}\right]^{T}
$$

Parameters of this vector are computed again from the differential equation

$$
y_{\delta}(k)=\boldsymbol{\theta}_{\delta}^{T}(k) \cdot \boldsymbol{\varphi}_{\delta}(k-1)+e(k)
$$

for $e(k)$ as a general random immeasurable component.

\subsection{Identification Method}

The last what needs to be described is the online identification method which satisfies the adaptivity of the controller. The Recursive Least-Squares Method [14] could be used because it is simple, accurate with modifications and it is also easily programmable.

Presented method could be used for both CT and DT identification models described above. The RLS method used for estimation of vectors of parameters $\hat{\boldsymbol{\theta}}_{C T}^{T}$ or $\hat{\boldsymbol{\theta}}_{\delta}^{T}$ in (24) or (30) could be described generally by the set of equations:

$$
\begin{gathered}
\varepsilon(k)=y(k)-\boldsymbol{\varphi}^{T}(k) \cdot \hat{\boldsymbol{\theta}}(k-1) \\
\gamma(k)=\left[1+\boldsymbol{\varphi}^{T}(k) \cdot \mathbf{P}(k-1) \cdot \boldsymbol{\varphi}(k)\right]^{-1} \\
\boldsymbol{L}(k)=\gamma(k) \cdot \mathbf{P}(k-1) \cdot \boldsymbol{\varphi}(k) \\
\mathbf{P}(k)=\frac{1}{\lambda_{1}(k-1)}\left[\begin{array}{c}
\left.\mathbf{P}(k-1)-\frac{\mathbf{P}(k-1) \cdot \boldsymbol{\varphi}(k) \cdot \boldsymbol{\varphi}^{T}(k) \cdot \mathbf{P}(k-1)}{\frac{\lambda_{1}(k-1)}{\lambda_{2}(k-1)}+\boldsymbol{\varphi}^{T}(k) \cdot \mathbf{P}(k-1) \cdot \boldsymbol{\varphi}(k)}\right] \\
\hat{\boldsymbol{\theta}}(k)=\hat{\boldsymbol{\theta}}(k-1)+\boldsymbol{L}(k) \varepsilon(k)
\end{array}\right.
\end{gathered}
$$

where $\phi$ is regression vector, $\varepsilon$ denotes a prediction error, $\mathbf{P}$ is a covariance matrix and $\lambda_{1}$ and $\lambda_{2}$ are forgetting factors. For example constant exponential forgetting [14] uses $\lambda_{2}=1$ and

$$
\lambda_{1}(k)=1-K \cdot \gamma(k) \cdot \varepsilon^{2}(k)
$$

where $K$ is a very small value (e.g. $K=0.001$ ). This RLS modification was used in this work for the online estimation.

\section{Simulation Experiment}

Proposed adaptive controller with two identification models was tested by simulation on the mathematical model of CSTR presented in Chapter 2.

Due to comparability, both simulations were performed for the same simulation parameters. The sampling period was $T_{v}=0.3 \mathrm{~min}$, the initial covariance matrix $\mathbf{P}(0)$ has on the diagonal $1 \cdot 10^{6}$ and starting vectors of parameters for the identification was chosen $\boldsymbol{\theta}_{C T}(0)=\boldsymbol{\theta}_{C T}(0)=[0.1 ; 0.1 ; 0.1 ; 0.1]^{T}$. The simulation was performed for $750 \mathrm{~min}$ and there were done 5 changes of the reference signal $w(t)$ during this time.

The controller needs some time for adaptation and our previous experiments have shown that it is good to insert the first change of the reference signal as an exponential function instead of the step function. The next changes were step functions The input signal $u(t)$ was limited to the values $u(t)=<-75 \% ;+75 \%>$ due to physical limitations. 
As it was mentioned, the tuning parameter for this adaptive controller is the position of the root $\alpha$. There were observed courses of the output variable y for three values of $\alpha=0.05 ; 0.08$ and 0.4 for both identification models and results are shown in the following figures.

The first analysis was done for the CT identification model where the filtered polynomial $c(\sigma)$ was $c(\sigma)=s^{2}+1.4 s+0.49$.

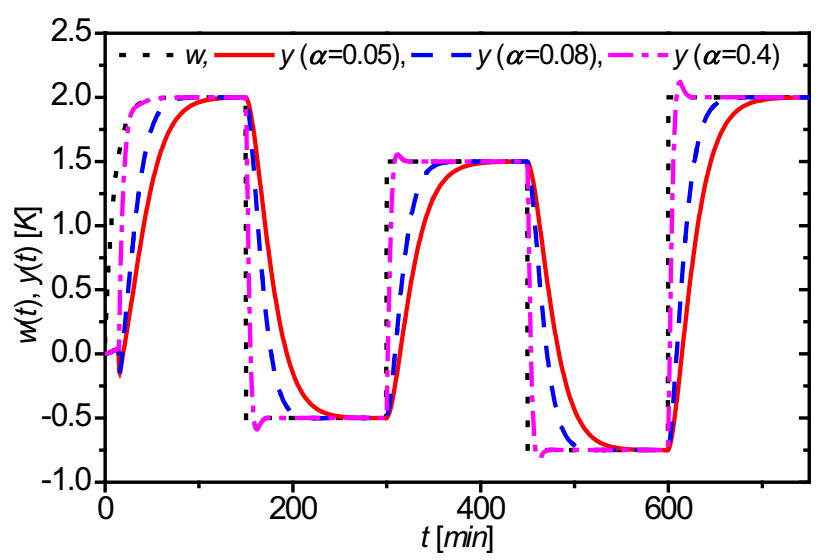

Figure 4. The course of the reference signal $w(t)$ and the output variable $y(t)$ for CT identification model and various parameter $\alpha$

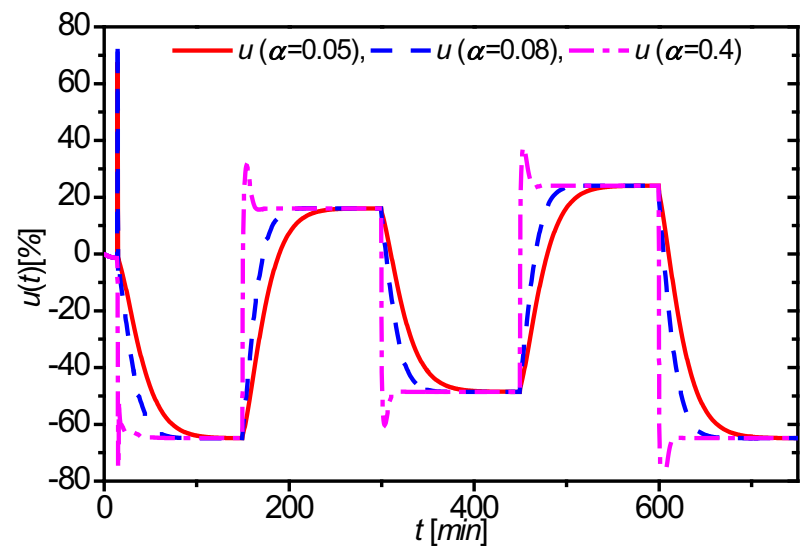

Figure 5. The course of the the input variable $u(t)$ for CT identification model and various parameter $\alpha$

Results show that the tuning parameter $\alpha$ affect mainly the speed of the control. Increasing value of $\alpha$ produces quicker output response but could end with small overshoots which is evident for $\alpha=0.4$. On the other hand, a smaller value of $\alpha$ has smoother course of the input variable $u(t)$ in Figure 5 which is better from practical point of view.

The second analysis for DT delta model was performed for Forward delta model and the same tuning parameters $\alpha$.

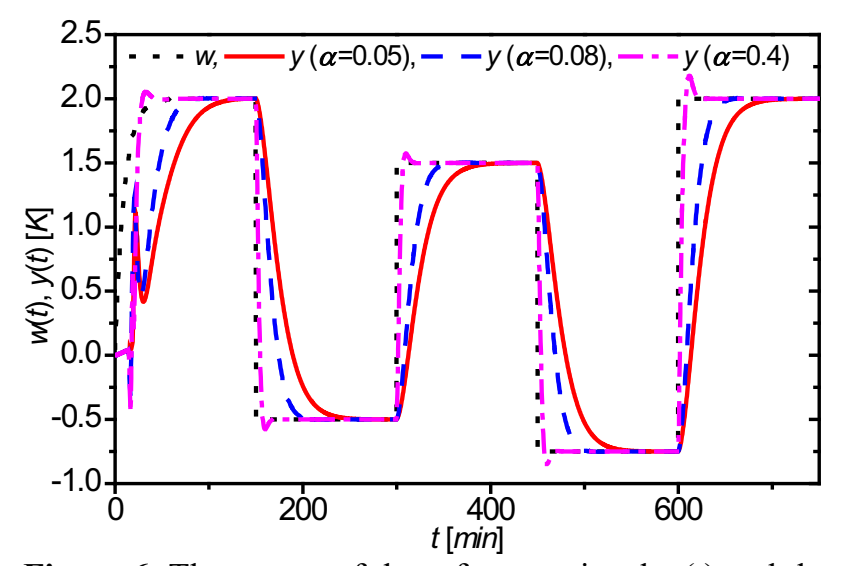

Figure 6. The course of the reference signal $w(t)$ and the output variable $y(t)$ for delta identification model and various parameter $\alpha$

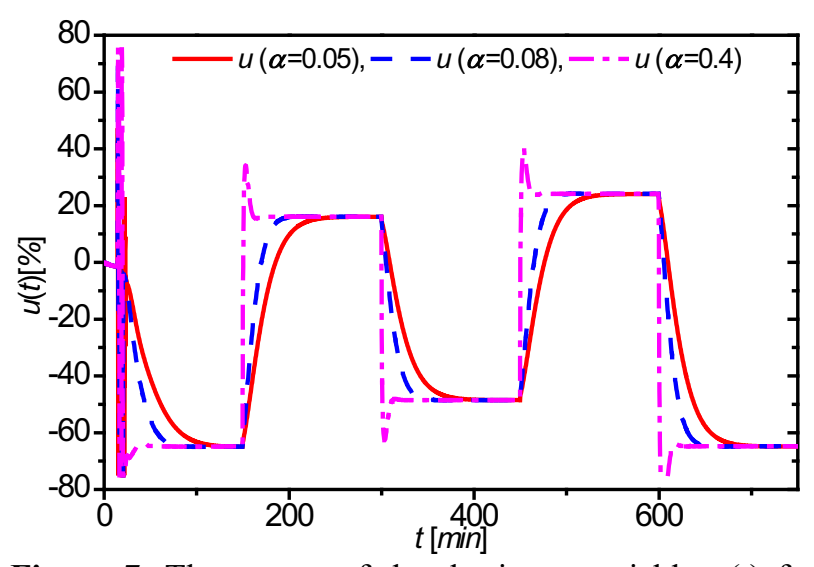

Figure 7. The course of the the input variable $u(t)$ for delta identification model and various parameter $\alpha$

Obtained results are very similar to those in the previous analysis. The biggest value of $\alpha=0.4$ has again the quickest course but overshoots and their value depends on the height of the change. The course of the input variable is smoother for the lower values of tuning parameter.

Both studies have very good control results except the beginning of the control. This is caused by the inaccurate identification which starts from the general point and it needs some time for "adaptation". On the other hand, after initial $50 \mathrm{~min}$ the controller does not have problem with the online identification.

Courses of the identified parameters are in Figures 8 11. These figures show that recursive least squares method used for identification has no problem with the identification except the beginning of the control in the adaptation part. 


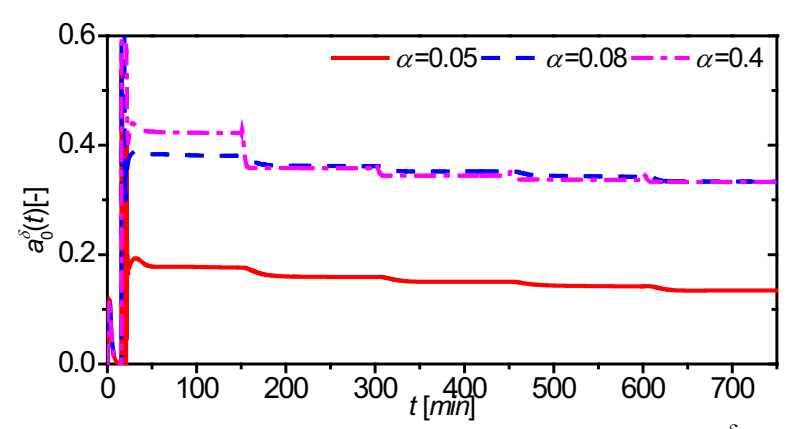

Figure 8. The course of the identified parameter $a_{0}{ }^{\delta}(\mathrm{t})$ for various $\alpha$ in delta identification model

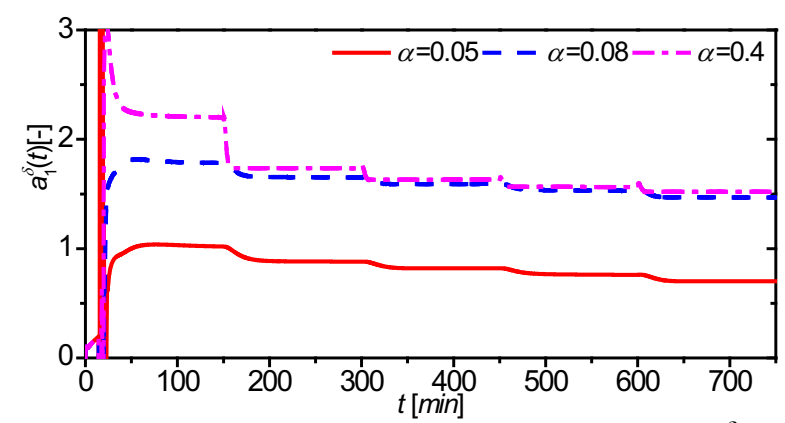

Figure 9. The course of the identified parameter $a_{1}{ }^{\delta}(\mathrm{t})$ for various $\alpha$ in delta identification model

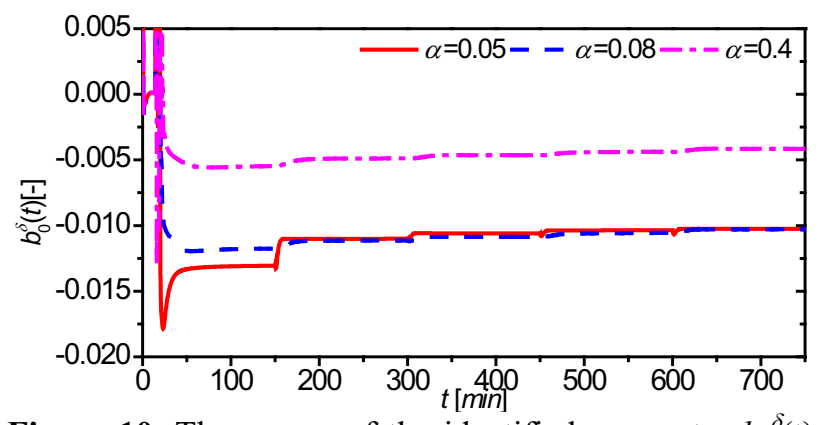

Figure 10. The course of the identified parameter $b_{0}{ }^{\delta}(\mathrm{t})$ for various $\alpha$ in delta identification model

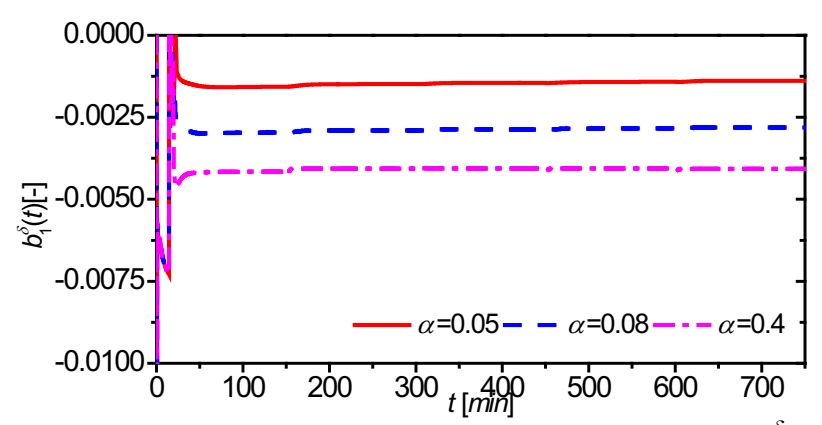

Figure 11. The course of the identified parameter $b_{1}{ }^{\delta}(\mathrm{t})$ for various $\alpha$ in delta identification model

\section{Conclusions}

The paper shows one approach for controlling of the nonlinear process represented by the continuous stirredtank reactor with the cooling in the jacket. The mathematical model of this reactor is described by the set of four nonlinear ODE that are easily solvable by the numerical methods. Proposed control strategy is based on the choice of the ELM parameters of which are identified recursively during the control and parameters of the controller are recomputed according to the identified ones. This controller could be tuned by the parameter $\alpha$ as a position of the root in the pole-placement method. The simulation experiments have shown that the increasing value of this parameter affect the speed of the control and overshoots - bigger value of $\alpha$ results in quicker output response but overshoots. Proposed controller produces good control results although this system has nonlinear behaviour and negative properties from the control point of view.

\section{References}

1. J. Ingham, I. J. Dunn, E. Heinzle, J. E. Prenosil. Chemical Engineering Dynamics. An Introduction to Modeling and Computer Simulation. Second. Completely Revised Edition. (VCH Verlagsgesellshaft. Weinheim, 2000).

2. K. J. Åström, B. Wittenmark 1989. Adaptive Control. (Addison Wesley. Reading. MA, 1989).

3. V. Bobal, J. Böhm, J. Fessl, J. Machacek. Digital Self-tuning Controllers: Algorithms. Implementation and Applications. Advanced Textbooks in Control and Signal Processing. (Springer-Verlag London Limited. 2005).

4. B. Wahlberg,., Automatica, vol. 26, 167-170 (1990).

5. R. H. Middleton, G. C. Goodwin. Digital Control and Estimation - A Unified Approach. (Prentice Hall. Englewood Cliffs, 2004).

6. D. L. Stericker, N. K. Sinha. Control-Theory and Advanced Technology. vol. 9. 113-125 (1993).

7. J. Vojtesek, P. Dostal. Proceedings of 19th European Conference on Modelling and Simulation ECMS 2005. Riga, Latvia, p. 591-598 (2005).

8. J. Vojtesek, P. Dostal. Proceedings 25th European Conference on Modelling and Simulation ECMS 2011. Nicosia: p. 501-507. ISBN 978-0-9564944-29 (2011).

9. H. Chen, A. Kremling; F. Allgöwer. Proceedings of 3rd European Control Conference. Rome, Italy (1995).

10. V. Kucera. Automatica. 29. 1361-1375 (1993).

11. M. J. Grimble Robust industrial control. Optimal design approach for polynomial systems. (Prentice Hall, London, 1994).

12. P. Dostal, V. Bobal; M. Blaha, M. Proceedings of IFAC Workshop on Adaptation and Learning in Control and Signal Processing ALCOSP 2001, 407412, Cernobbio-Como, Italy, (2001).

13. S. Mukhopadhyay, A. G. Patra, G. P. Rao. International Journal of Control, vol.55, 1161-1187 (1992).

14. J. Mikleš, M. Fikar, Process modellig, identification and control 2 (STU Press, Bratislava, 2004). 